\title{
Kinetic equation for solitons in sine-Gordon and nonlinear Schrödinger equations
}

\author{
I.V.Baryakhtar \\ Institute for Low Temperature Physics and Engineering, \\ 47 Lenin Ave., 310164 Kharkov, Ukraine
}

Received June 29, 1998

The scheme for describing the kinetic behaviour of solitons in the integrable systems is considered for sine-Gordon and nonlinear Schrödinger equations. The collision integral for solitons is constructed without the assumption of the gas approximation. The kinetic equation for solitons in the case of small inhomogeneities in the soliton gas is proposed. Possible applications of the kinetic properties of solitons are discussed.

Key words: soliton, collision integral, entropy

PACS: 05.20.Dd

\section{Introduction}

Soliton is one of the most actively investigated nonlinear objects. Soliton ideas were successfully applied for explaining the physical effects in solids, hydrodynamics, plasma $[1,2]$ and biological systems [3]. One soliton solution of corresponding equations has been used in the most of soliton applications. However, in the integrable systems soliton-soliton interaction demonstrates a very important property: solitons conserve their velocities and therefore energies and get only the shifts of their positions (see for example [1]). This fact enables us to expect unusual kinetic behaviour of solitons. First, kinetic properties of solitons were considered by Zakharov [4]. He has constructed kinetic equation for solitons in Korteweg - de Vries equation, but this equation describes only renormalization of solitons velocities and does not describe the diffusion processes. From the other point of view kinetic behaviour was considered by Wada and Schrieffer [5] and Theodorakopoulos [6]. In [5,6] the diffusion of kinks interacting with phonons in $\varphi^{4}$ model was considered. This model is not exactly an integrable model but potential for kink-phonons interaction has a reflectionless form and the result of the interaction is the same as for soliton-soliton collision in integrable models. Taking into account such kind of interaction in $[5,6]$ kink diffusion coefficient was calculated. A bit later the same calculations have been 
done for sine-Gordon model [7]. Following the approach [5,6], diffusion coefficient and renormalization of kink velocity due to kink-breather collision were calculated in sine-Gordon model in [8]. In fact the regular method of investigating the soliton kinetic properties has not been formulated. The problem of the entropy of the system of solitons was considered in [9] and it was shown that entropy increases in the case of inhomogeneous distribution of solitons in real space on the example of kinks in sine-Gordon equation. It enables us to derive the transport equations for kinks and to prove the existence of other kinetic coefficients, except the diffusion coefficient for the kinks in sine-Gordon equation. In [10] the kinetic equation for solitons interaction with $2 \mathrm{D}$ phonons in the system close to the completely integrable one has been proposed.

In the present paper the scheme for describing the kinetic behaviour of solitons is considered for sine-Gordon and nonlinear Schrödinger equations. We follow the Boltzmann approach for usual particles. The steps are the following: to analyze the processes of soliton-soliton interaction; to introduce the distribution function of solitons and to construct the collision integrals; to formulate the kinetic equation for solitons and to prove the entropy production; to calculate the corresponding kinetic coefficients and relaxation times.

\section{Models}

Among few physically reasonable models, covered by the inverse scattering method and described solitons we consider sine-Gordon (SG) and nonlinear Schrödinger (NS) models.

SG equation can be written as

$$
\frac{\partial^{2} u}{\partial t^{2}}-\frac{\partial^{2} u}{\partial t^{2}}+\omega_{0}^{2} \sin u=0
$$

SG equation is widely applied in analysing the nonlinear properties of magnets and Josephson junction $[1,3]$.

Two types of localized solutions are known for this equation: kink type soliton and breather type soliton. Kink is characterized by its coordinate of the centre of mass $x_{k}$ and its velocity $v_{k}$, breather - by the coordinate of the centre of mass $x_{b}$, the phase $\varphi_{b}$, the velocity $v_{b}$ and the intrinsic frequency $\omega_{b}$. When $\omega_{b} \rightarrow 1$ breather can be interpreted as a bound state of two kinks. Kink is the topological soliton, the topological charge of breather is equal to zero.

NS equation in dimensionless variables has the form:

$$
\mathrm{i} \frac{\partial \psi}{\partial t}+\frac{\partial^{2} \psi}{\partial x^{2}}+2 \psi|\psi|^{2}=0
$$

NS solitons, often named as envelope solitons, are characterized by the coordinate of the $\mathrm{v}$ centre of mass $x_{e}$, the velocity $v_{e}$, the phase $\varphi_{e}$ and the frequency $\omega_{e}$ NS soliton application see, for example, in [1-3]. 
In the integrable systems solitons clearly display the difference from the usual particles and waves in soliton-soliton collisions. Solitons conserve their forms and velocities and the only result of the collision is shifts of the soliton's coordinates and phases for breathers and envelope solitons [1]. It is necessary to emphasize that many particle effects are missing, i.e. the total shift of soliton position is equal to the sum of partial shifts [1].

The following formulae can be written for the two soliton collisions. For kink-kink collision

$$
\begin{aligned}
& v_{1, k}=v_{1, k}^{\prime}, \quad v_{2, k}=v_{2, k}^{\prime}, \\
& x_{1, k}^{\prime}=x_{1, k}+\Delta x_{1, k}\left(v_{1}, v_{2}\right), \quad x_{2, k}^{\prime}=x_{2, k}-\Delta x_{1, k}\left(v_{1}, v_{2}\right) .
\end{aligned}
$$

For breather-breather and envelope soliton-soliton collisions

$$
\begin{aligned}
& v_{1, i}=v_{1, i}^{\prime}, \quad v_{2, i}=v_{2, i}^{\prime}, \\
& x_{1, i}^{\prime}=x_{1, i}+\Delta x_{1, i}\left(v_{1}, v_{2}, \omega_{1}, \omega_{2}\right), \quad x_{2, i}^{\prime}=x_{2, i}-\Delta x_{2, i}\left(v_{1}, v_{2}, \omega_{1}, \omega_{2}\right) ; \\
& \omega_{1, i}=\omega_{1, i}^{\prime}, \quad \omega_{2, i}=\omega_{2, i}^{\prime}, \\
& \varphi_{1, i}^{\prime}=\varphi_{1, i}+\Delta x_{1, i}\left(v_{1}, v_{2}, \omega_{1}, \omega_{2}\right), \quad \varphi_{2, i}^{\prime}=\varphi_{2, i}-\Delta x_{2, i}\left(v_{1}, v_{2}, \omega_{1}, \omega_{2}\right) .
\end{aligned}
$$

Here $i=b, e$ for breather and envelope soliton; the values after collision are noted with a prime. The general expression for coordinate and phase through the scattering date see, for example, in [1].

\section{Kinetic equations and entropy production}

Let us introduce distribution function of solitons

$$
f_{i}\left(\Gamma_{i}, t\right), \quad i=k, b, e ;
$$

where $\Gamma_{k}=(x, v)$ for kinks, $\Gamma_{i}=(x, v, \varphi, \omega)$ for breathers and envelope solitons. Following the Boltzmann way the collision integral can be written as

$$
\begin{aligned}
L\{f\}=\int & {\left[W\left(\Gamma_{1, i}, \Gamma_{2, l} ; \Gamma_{1, i}^{\prime}, \Gamma_{2, l}^{\prime}\right) f_{i}\left(\Gamma_{1, i}^{\prime}, t\right) f_{l}\left(\Gamma_{2, l}^{\prime}, t\right)\right.} \\
& \left.-W\left(\Gamma_{1, i}^{\prime}, \Gamma_{2, l}^{\prime} ; \Gamma_{1, i}, \Gamma_{2, k}\right) f_{i}\left(\Gamma_{1, i}, t\right) f_{l}\left(\Gamma_{2, l}, t\right)\right] \mathrm{d} \Gamma_{1, i}^{\prime} \mathrm{d} \Gamma_{2, l}^{\prime} \mathrm{d} \Gamma_{2, l},
\end{aligned}
$$

where $W$ is probability of soliton transition per unit time.

For simplicity we will consider only the changes of soliton coordinates. Taking into account the fact that the cross section of soliton-soliton collision is equal to 1 due to the one dimensionality of the problem, $W$ can be written as

$$
W=1 \cdot\left|v_{1, i}-v_{2, l}\right| \delta\left(v_{1, i}-v_{1, i}^{\prime}\right) \delta\left(v_{2, l}-v_{2, l}^{\prime}\right) \delta\left(x_{1, i}^{\prime}-x_{1, i}-\Delta x_{1, i}\right) \delta\left(x_{2, l}^{\prime}-x_{2, l}-\Delta x_{2, l}\right) .
$$

Here the first two delta functions describe the solitons momentum conservation laws, the two last delta functions describe the fact of solitons shifts due to the collision. 
It is necessary to underline that the gas approximation has not been used in (6), (7) because many particle effects for solitons are missing.

Assuming that $f(x, V, t)$ is slow varying in scales comparing to $\Delta x$ and expanding $f(x, V, t)$ in powers of $\Delta x$ and keeping the leading terms we can rewrite the expression for collision integral (6) in the form:

$$
L\left\{f_{i}\right\}=-\delta v_{1, i} \frac{\partial f_{i}}{\partial x}+D_{1, i} \frac{\partial^{2} f_{i}}{\partial x^{2}}
$$

where

$$
\delta v_{1, i}=\int\left|v_{1, i}-v_{2, l}\right| \Delta x_{1, i}\left(V_{1, i}, V_{2, l}\right) f_{i}\left(x_{2, l}, V_{2, l}, t\right) \mathrm{d} \Gamma_{2, l}
$$

is the renormalization of soliton velocity due to collision and

$$
D_{1, i}=\int\left|v_{1, i}-v_{2, l}\right|\left(\Delta x_{1, i}\left(V_{1, i}, V_{2, l}\right)\right)^{2} f_{i}\left(x_{2, l}, V_{2, l}, t\right) \mathrm{d} \Gamma_{2, l}
$$

describes the soliton diffusion process. Here $V_{i}=v_{k}$ for kinks and $V_{i}=\left(v_{i}, \omega_{i}\right)$ for breathers and envelope solitons.

The kinetic equation can be written as

$$
\frac{\partial f_{i}}{\partial t}+\left(v+\delta v_{1, i}\right) \frac{\partial f_{i}}{\partial x}=D_{1, i} \frac{\partial^{2} f_{i}}{\partial x^{2}}
$$

From kinetic equation one can show that soliton-soliton collisions lead to entropy production in kink gas. Really, defining entropy as

$$
S=-\int f\left(x, V_{1, i}, t\right) \ln \left[f\left(x, V_{1, i}, t\right) / e\right] \mathrm{d} \Gamma_{1, i}
$$

and using kinetic equation and formula (10) one can obtain that

$$
\frac{\mathrm{d} S}{\mathrm{~d} t}=\int q \mathrm{~d} \Gamma_{1, i}
$$

where the source of entropy production is:

$$
q=\int\left|v_{1, i}-v_{2, l}\right|\left[\Delta x_{1, i}\left(V_{1, i}, V_{2, l}\right)\right]^{2} \frac{f_{i}}{f_{l}} \quad\left(\frac{\partial f_{i}}{\partial x}\right)^{2} \mathrm{~d} \Gamma_{2, l} \geqslant 0 .
$$

The expression (14) proves the Boltzmann entropy production theorem. The entropy production connect only with the inhomogeneities in real space. In addition, when the inhomogeneities in the phase space take place, the entropy production can be shown similarly.

Applying the standard methods from (11) it is easy to derive the transport equations and to calculate kinetic coefficients. The transport equations can be written in the following general form:

$$
\frac{\partial}{\partial t} n_{i}<a_{i}>+\frac{\partial}{\partial x}\left(j_{i}+j_{i}^{d}\right)=0
$$


where $n_{i}$ is density of i-type soliton and

$$
\begin{aligned}
n_{i}<a_{i}> & =\int a_{i}\left(x, V_{i}\right) f\left(x, V_{i}, t\right) \mathrm{d} V_{i}, \\
j_{i} & =\int a_{i}\left(x, V_{i}\right)\left[v+\delta v_{i}\right] f_{i}\left(x, V_{i}, t\right) \mathrm{d} V_{i}, \\
j_{i}^{d} & =-\frac{\mathrm{d}}{\mathrm{d} x} \int a_{i}\left(x, V_{i}\right) D_{i} f_{i}\left(x, V_{i}, t\right) \mathrm{d} V_{i} .
\end{aligned}
$$

Substituting in equation (1), the velocity and the energy of solitons for variable $a$, one can obtain the continuity equation, hydrodynamic equation and equation for local energy density.

To calculate the specific kinetic coefficients it is necessary to assume the distribution function of solitons. For kinks in sine-Gordon equation in the case of Maxwell-Boltzmann distribution function, the self-diffusion, thermal conductivity and internal friction coefficients were calculated in [9].

\section{Applications}

Let us consider some applications that result from kinetic equation (11). NS equation appears in the analysis of nonlinear electron plasma waves $[1,2,11]$. The conditions of solitons creation [12] and collapse [13] and interpretation of Lengmuir turbulence in soliton terms have been analyzed in the frame of NS equation (see [11]).

In the nonintegrable system solitons collide with momentum changing, but in the case close to completely integrable model it is not difficult to estimate the relaxation time due momentum exchange, $1 / \tau_{\text {ex }} \sim \Delta E / E$, where $\Delta E / E$ is relative energy change due to collision. Thus we have two steps of the relaxation: the first one deals with shifts of the soliton positions and the second deals with momentum exchange [10]. The characteristic time $\tau_{\text {in }}$ of soliton instability in $2 \mathrm{D}$ space is finite and $\tau_{\text {ins }} \sim L^{2}$ (see [11]), where $\mathrm{L}$ is a characteristic size in the direction perpendicular to soliton propagation. In other words it is possible to conclude that the above considered kinetic behaviour of solitons deals with shift of its positions and can be realized as an intermediate regime before solitons collapse and can be interpreted as soliton turbulence. Soliton turbulence phenomena have been considered in [2], where kinetic equation for many particle distribution function of soliton like waves has been proposed. In fact in [2] processes with momentum exchange only were considered.

Other application of NS equation deals with soliton propagation in optic fibers [14]. From the applied point of view the problem of relaxation of solitons interacting with defects is one of the most urgent among the kinetic effects. The possible results of soliton-defect interaction are transmission, reflection or capturing of soliton by defect (see [15]). Dynamic properties of solitons in optic fiber interacting with dispersion-spectrum inhomogeneities have been studied in [16]. Obviously, the most natural way of analyzing the relaxation of solitons is to formulate soliton-defect 
collision integral. In general form it can be written as

$$
L_{\mathrm{sd}}=\int W\left(p_{1}, p_{2}\right)\left[f\left(x, p_{1}, t\right)-f\left(x, p_{2}, t\right)\right] \mathrm{d} p_{2} .
$$

A specific expression for $W\left(p_{1}, p_{2}\right)$ in sine-Gordon model was calculated in [17] for the case of elastic scattering of soliton by impurity.

Here we estimate the relaxation time $\tau_{\text {sd }}$ in the case of low density of solitons comparing with defects concentration $C_{\mathrm{d}}$. Really from dimensional consideration

$$
\frac{1}{\tau_{\mathrm{sd}}} \sim C_{\mathrm{d}} \frac{\Delta E_{\mathrm{sd}}}{E}
$$

where $\Delta E_{\mathrm{sd}}$ is a relative energy loss due to interaction with defect.

The presense of defects can be induced by fiber irradiation. As a result, the characteristics of light and sound propagation have changed under the influence of the ionizing radiation. These effects have been applied for measuring the radiation dose $[18,19]$. Obviously, the relaxation time and kinetic coefficients of solitons depend on the intensity of fiber irradiation.

Let us consider the expression for the concentration of defects in the case of $\gamma$-rays. The $\gamma$-rays can be generated in the processes of electron beam breaking on heavy elements targets. The breaking radiation has a continuous spectrum with the maximum energy of photons equal to the energy of electrons (see for example [20]). In this case [21]

$$
C_{\mathrm{d}}=J_{\mathrm{e}} K t
$$

where $\mathrm{t}$ is the time of irradiation, $\mathrm{J}$ is the current of electrons, coefficient $\mathrm{K}=2.1$ for Si [21] and energy of $\gamma$-rays $E_{\gamma} \geqslant 20 \mathrm{Mev}$. Therefore

$$
J_{\mathrm{e}} \sim \frac{1}{\tau_{\mathrm{sd}} t\left(\Delta E_{\mathrm{sd}} / E\right)} .
$$

Consequently due to the stability of soliton and low damping in optical fiber this effect can be applied for measuring the irradiation dose.

\section{Acknowledgements}

The author is grateful to V.G.Baryakhtar and E.N.Economou for discussions. This work was supported by INTAS grants No 93-1324 and No 94-3754, and by International Atomic Energy Agency, contract N 7996.

\section{References}

1. Zakharov V.E., Manakov S.V., Novikov S.P., Pitaevskii L.P. Theory of Solitons, Moscow, Nauka, 1980 (in Russian).

2. Zaslavskii G., Sagdeev R.Z. Introduction to Nonlinear Physics. Moscow, Nauka, 1988 (in Russian). 
3. Davydov A.S. Solitons in Molecular Systems. Kiev, Naukova Dumka, 1988 (in Russian).

4. Zakharov V.E. Kinetic equation for solitons. // Zh. Eksp. Theor. Fiz., 1971, vol. 60, p. 993-1000 (in Russian).

5. Wada Y., Schrieffer J.R. Brownian motion of a domain wall and the diffusion constants. // Phys. Rev. B, 1978, vol. 18, p. 3897-3912.

6. Theodorakopoulos N. Dynamics of nonlinear systems: the kink-phonon interaction // Z. Phys. B, 1979, vol. 33, p. 385-390.

7. Fesser K. Stochastic motion of sine-Gordon-solitons and the spin-correlation function // Z. Phys. B, 1979, vol. 39, p. 47-52.

8. Sasaki K., Maki K. Soliton dynamics in a magnetic chain. I. Antiferromagnet. // Phys. Rev. B, 1987, vol. 35, p. 257-262.

9. Baryakhtar I.V., Baryakhtar V.G., Economou E.N. Kinetic equation and transport coefficients for kink solitons in the sine-Gordon equation. Phys. Lett. A, 1985, vol. 207, p. $67-71$.

10. Baryakhtar I.V. On the kinetic equation for solitons interaction with phonons. Preprint ILT-1-96, Kharkov, 1996, 8 p.

11. Kadomtsev B.B. Collective Phenomena in Plasma, 2nd edn., Moscow, Nauka, 1988 (in Russian).

12. Vedenov A.A., Rudakov L.I. Wave interaction in continuous media // Doklady AN, 1964, vol. 159, p. 767-770 (in Russian).

13. Zakharov V.E. Collaps of Langmuir waves. // Zh. Eksp. Theor. Fiz., 1972, vol. 62, p. 1747-1759 (in Russian).

14. Hasegawa A., Tappert F. Transmission of stationary optical pulses. // Appl. Phys. Lett., 1973, vol. 23, p. 142-144.

15. Bass F.G., Kivshar Yu.S., Konotop V.V., Sinitsin Yu.A. Dynamics of solitons under random perturbations. // Phys. Reports, 1988, vol. 157, p. 63-181.

16. Vysloukh V. A., Serkin V. N., Danilenko A. Yu., Samarina E. V. Influence of the dispersion-spectrum inhomogeneities. // Quantum Electronics, 1995, vol. 22, p. 11291134.

17. Baryakhtar I.V., Baryakhtar V.G., Economou E.N. Additivity of diffusion coefficients for solitons. // Europhys. Lett, 1996, vol. 36(2), p. 87-91.

18. Vassilopoulos C., Kortis A., Mantakas C., Conception of an ionising radiation detection scheme. // IEE Proc. J, 1993, vol. 140, p. 267-272.

19. Poret J.C., Lindgren E., Rosen M., Suter J.J., Rifkind J. M. Laser-generated ultrasonic characterization of irradiated glass optical fibers. // J. Non-Cryst. Solids, 1993, vol. 160 , p. $82-88$.

20. Kovalev V.P. Secondary Irradiation of Electron Accelerator. Moscow, Energoatomizdat, 1979 (in Russian).

21. Zablotskii V.V., Ivanov I.A., Kosmach V.F., Leonov N.N., Ostroumov V.I. Doping of semiconductors by photonuclear reactions. // Fiz. Tech. Poluprovod., 1986, vol. 20, p. 625-629 (in Russian). 


\section{Кінетичне рівняння для солітонів у моделі синус-Гордона та нелінійній моделі Шредінгера}

\section{І.В.Бар'яхтар}

Фізико-технічний інститут низьких температур ім. Б.І.Вєркіна НАН України, 310164 Харків, просп. Леніна, 47

Отримано 29 червня 1999 р.

Пропонується схема опису кінетичної поведінки солітонів у інтегровних системах на прикладі моделі синус-Гордона та нелінійної моделі Шредінгера. Побудовано інтеграл зіткнень, що не використовує газове наближення для солітонів. Пропонується кінетичне рівняння для солітонів у випадку невеликої неоднорідності. Обговорюється можливе використання кінетичних властивостей солітонів.

Ключові слова: солітон, ентропія

PACS: 05.20.Dd 